\title{
EchoGéo
}

$8 \mid 2009$

Moyen-Orient : conflits et mobilités dans un espace mondialisé

\section{Jérusalem, entre attraction et répulsion migratoire}

Irène Salenson

\section{(2) OpenEdition}

Journals

Édition électronique

URL : https://journals.openedition.org/echogeo/10932

DOI : 10.4000/echogeo. 10932

ISSN : 1963-1197

Éditeur

Pôle de recherche pour l'organisation et la diffusion de l'information géographique (CNRS UMR 8586)

Référence électronique

Irène Salenson, « Jérusalem, entre attraction et répulsion migratoire », EchoGéo [En ligne], 8 | 2009, mis en ligne le 04 mars 2009, consulté le 31 juillet 2021. URL : http://journals.openedition.org/echogeo/ 10932 ; DOI : https://doi.org/10.4000/echogeo.10932

Ce document a été généré automatiquement le 31 juillet 2021.

EchoGéo est mis à disposition selon les termes de la licence Creative Commons Attribution - Pas d'Utilisation Commerciale - Pas de Modification 4.0 International (CC BY-NC-ND) 


\title{
Jérusalem, entre attraction et répulsion migratoire
}

\author{
Irène Salenson
}

1 De nos jours, Jérusalem fait fuir, Jérusalem perd des habitants, qu'il s'agisse de populations israélienne ou palestinienne. On pourrait s'étonner de cette tendance négative dans une ville considérée comme sainte pour les trois religions monothéistes, chargée d'une puissance symbolique à l'échelle internationale et capitale hautement revendiquée par deux mouvements nationaux antagonistes. Jérusalem ne serait donc pas aussi attractive qu'on peut l'imaginer...

2 Qui plus est, le solde migratoire est de plus en plus déficitaire depuis le début des années 1990, pour Jérusalem-Ouest comme pour Jérusalem-Est: entre 1968 et 1988, le solde tournait autour de 600 personnes en moins par an, avec des pics de 1000 à 2000 par an au tournant des années 1980, lorsque la construction des implantations satellites de l'agglomération drainait des foyers hiérosolomitains (Maale Adumim, Givat Zeev, Mevasseret Zion.... ${ }^{1}$. Depuis 1990, le solde migratoire s'établit en moyenne à hauteur de 5000 départs par an, avec un maximum atteint en 2000 (-8 200 personnes). 


\section{Jèrusalem / Al Quds : une ville aux limites complexes}

La $\alpha$ vieille ville * de Jérusalem, ceinte par des remparts médiévaux qui ont contenu l'extension urbaine jusqu'à la fin du XIX $X^{e}$ siècle, possède une superficie de moins $\mathrm{d}^{\prime} 1 \mathrm{~km}^{2}$, tandis que le périmètre municipal actuel, défini de façon unilatérale par Israēl depuis 1967, s'étend sur $126 \mathrm{~km}^{2}$. Du côté palestinien, un périmètre de référence historique est celui de la municipalité de répoque jordanienne (1948-1967), qui couvrait $6 \mathrm{~km}^{2}$ seulement. correspondant aujourd'hui aux quartiers centraux de la Jérusalem arabe, c'est-â-dire la vieille ville et ses alentours. Mais l'Autorité nationale palestinienne (ANP), créée en 1994, a divisé la Cisjordanie en 12 gouvernorats, qui reprennent en partie les découpages administratifs jordaniens. Dans ce découpage, la superficie du gouvernorat de Jérusalem représente à peu près cinq fois le périmètre de la Jérusalem municipale israélienne. Ce périmètre est lui-même subdivisé par l'Autorité palestinienne entre la zone située à l'intérieur du domaine municipal israélien, dénommée $\alpha \mathrm{J1}$ et le reste du territoire du gouvernorat, situé en Cisjordanie et dénommé a J2 \%. L'ANP ne peut pas gouverner à Jérusalem-Est (J1), car cet espace est sous contrôle israélien depuis 1967. Elle reconnaît donc de facto le découpage frontalier israèlien (notamment pour le recensement et pour la production de données statistiques), bien qu'elle le considère comme illegitime et arbitraire.

La population palestinienne est plus nombreuse à l'intérieur de la zone $\mathrm{J} 1$ que dans le reste du gouvernorat de Jérusalem, qui couvre des espaces désertiques. En 2006, selon le Bureau palestinien des statistiques, le gouvernorat comptait une population totale de 407000 habitants palestiniens, dont les deux tiers habitaient à Jérusalem-Est (250 000).

Si l'on ajoute la population de Jérusalem-Quest, on dénombre 720000 habitants dans le périmètre municipal israélien (470 000 Israéliens et 250000 Palestiniens).

C'est pourquoi cet article vise à décrypter l'origine et les caractéristiques des pertes migratoires subies par Jérusalem/Al Quds², en partant des données chiffrées produites par le Bureau israélien des statistiques (Israeli Central Bureau of Statistics) et le Bureau Palestinien des statistiques (Palestinian Central Bureau of Statistics) ${ }^{3}$.

Nous analyserons les causes de la difficulté de Jérusalem à retenir ses habitants, qu'ils soient israéliens ou palestiniens, en tentant de dépasser les idées reçues et de discuter parfois la présentation officielle des flux, en évoquant la possibilité de flux informels. Ce solde migratoire négatif continu trouve ses racines dans un ensemble de facteurs qu'il est possible de catégoriser tant en termes conjoncturels qu'à l'échelle des populations considérées. Si les motivations politiques jouent évidemment un rôle fondamental (concurrence pour l'occupation de l'espace entre Israéliens et Palestiniens), le solde migratoire négatif montre précisément que ceux-ci sont mêlés à des facteurs économiques et sociaux qui deviennent parfois aussi importants que les facteurs politiques pour expliquer les dynamiques migratoires affectant Jérusalem.

\section{Une répulsion qui s'accentue pour les Juifs israéliens}

5 En ce qui concerne la population juive israélienne, les pertes migratoires correspondent à une tendance lourde installée depuis 1967 et non à un retournement conjoncturel plus récent qui serait lié à une crise politique ou économique.

Dans les commentaires israéliens, notamment dans la presse, les facteurs généralement évoqués sont la situation sécuritaire instable et durable (liée au conflit israélopalestinien), la croissance de la population ultra-orthodoxe qui ferait fuir les « laïcs » et le manque d'emplois.

\section{Concurrence de Tel Aviv?}

Dans les représentations collectives israéliennes, la majorité des émigrants sont des jeunes cadres dynamiques, qui se dirigent vers l'agglomération de Tel Aviv, à la recherche d'un emploi plus qualifié et/ou mieux rémunéré qu'à Jérusalem ${ }^{4}$. 
8 La présentation du poids de chacune des deux villes par les instituts statistiques israéliens et par les publications gouvernementales officielles illustre elle-même la concurrence entre les deux agglomérations. En effet, ces publications rappellent régulièrement que Jérusalem est la première ville de l'Etat hébreu par son poids démographique : 720000 habitants en 2006, alors que Tel Aviv-Jaffa, la deuxième ville, ne compte que 375000 habitants 5 .

9 Ces chiffres cachent en réalité le fait que la zone métropolitaine de Tel Aviv constitue en réalité la première agglomération israélienne : les localités qui entourent Tel Aviv formant une conurbation de plus de 3 millions d'habitants en $2006^{6}$. Tel Aviv est la capitale économique du pays, elle regroupe les sièges des plus grandes entreprises israéliennes et internationales.

10 La mise en avant du rang démographique de Jérusalem à l'échelle nationale illustre le processus de compétition qui oppose la capitale politique et la capitale économique. En effet, Jérusalem a été désignée par le gouvernement israélien comme sa capitale en 1950 et cette position a été réaffirmée en 1967, après la conquête militaire de la partie orientale de la ville, puis en 1980 par la loi fondamentale sur Jérusalem7.

11 La tendance au départ de jeunes "laïcs » de Jérusalem vers Tel Aviv engendrerait la hausse de la proportion des personnes âgées et des ultra-orthodoxes ${ }^{8}$ dans la population hiérosolomitaine, processus qui a suscité l'invention d'un néologisme dans la langue hébraïque : hitharedut (orthodoxisation). L'orthodoxisation et la situation sécuritaire sensible de Jérusalem susciteraient à leur tour de nouveaux départs. Cette interprétation est relayée par les médias israéliens ${ }^{9}$.

12 Or, les statistiques nous apprennent que les émigrants hiérosolomitains se dirigent certes vers Tel Aviv, mais également vers plusieurs villes de l'« espace métropolitain $»^{10}$ de Jérusalem, qui comprend les villes de Bet Shemesh, Modiin, Mevasseret Zion.

De même, Jérusalem n'est pas une ville vieillissante : l'âge médian est de 23,4 ans en 2005 (25,4 pour la seule population juive), tandis qu'il s'élève à 33,8 ans à Tel Aviv et 36,8 à Haïfa.

14 Enfin, selon les enquêtes effectuées par le Jerusalem Institute for Israel Studies, seuls 7 \% des personnes qui partent mentionnent l'orthodoxisation de Jérusalem comme facteur répulsif ${ }^{11}$. Du reste, la proportion relative des ultra-orthodoxes augmente peu (autour de 30\%), car ceux-ci fournissent un important contingent parmi les émigrants. Le démographe Sergio Della Pergola prévoit que leur part passera de $29 \%$ en 1995 à $32 \%$ de la population hiérosolomitaine en 2020 : «Contrairement à la perception véhiculée par de nombreux observateurs, ce ne sont pas les quartiers les plus religieux qui connaîtront la plus forte croissance $»^{12}$.

15 Comment comprendre alors la croissance du déficit migratoire de Jérusalem? La démographe Maya Choshen confirme que plus de la moitié des émigrants sont des personnes d'âge actif (entre 25 et 40 ans); cependant, la composition structurelle de la population et l'indice élevé de fécondité conduisent au maintien d'une population jeune dans la ville ${ }^{13}$.

\section{Manque d'emplois ?}

16 La recherche d'un emploi semble être un réel facteur motivant les départs. A Jérusalem, $44,6 \%$ de la population de plus de 15 ans seulement occupe un emploi, alors que la 
moyenne israélienne est de $55,1 \%^{14}$. En revanche, la proportion de chômeurs est inférieure à la moyenne nationale : 7,6 \% des actifs à Jérusalem contre 9,7 \% en Israël en $2005^{15}$. L'économiste Abraham Friedman en déduit que de nombreux hiérosolomitains travaillent en-dehors du territoire municipal ${ }^{16}$. On peut alors imaginer que ces migrants pendulaires soient tentés à moyen terme de déménager hors de la ville pour se rapprocher de leur lieu de travail, externe à Jérusalem : ils alimenteraient ainsi les flux d'émigration.

L'offre d'emplois est-elle réellement insuffisante à Jérusalem? Le principal secteur d'activités est la fonction publique : $41 \%$ des salariés travaillent dans l'administration, l'éducation, la santé et les services sociaux, ce qui représente le double de la part des emplois publics à Tel Aviv ${ }^{17}$. En effet, le gouvernement israélien a installé le siège de la plupart des ministères et des institutions nationales dans la capitale. La distribution est inversée pour les domaines des finances et des affaires : $13 \%$ des actifs à Jérusalem contre $33 \%$ à Tel Aviv. Les secteurs liés au tourisme constituent une part importante de l'emploi à Jérusalem: $18 \%$ des hiérosolomitains travaillent dans le commerce, l'hôtellerie et la restauration. En revanche, l'industrie ne regroupe que $8,1 \%$ des emplois, la moitié de la moyenne israélienne : sont classées dans le secteur secondaire les activités d'édition et d'imprimerie, importantes à Jérusalem. Les autres principales activités industrielles sont l'agro-alimentaire, l'électronique et la production de matériaux métalliques.

Mais le nombre d'emplois dans l'industrie a baissé depuis les années 1980, et Abraham Friedman mentionne un risque de délocalisation des activités de pointe hors de Jérusalem, à cause d'un manque de compétitivité et de l'extension de quartiers ultraorthodoxes à proximité de deux zones industrielles (Romema et Givat Shaul) qui ferait, selon lui, fuir les entreprises ${ }^{18}$. Par ailleurs, les restrictions budgétaires gouvernementales ont entraîné des suppressions de postes dans la fonction publique. La capitale offre donc peu de nouvelles perspectives d'emplois.

\section{Les ultra-orthodoxes partent aussi}

19 Selon un sondage réalisé parmi des personnes ayant quitté la ville dans les années 1990, la cause de départ la plus fréquente était la question du logement ( $41 \%$ des réponses), c'est-à-dire la hausse des coûts immobiliers à Jérusalem, la volonté d'accéder à la propriété et/ou la recherche d'un logement plus grand ou d'un cadre de vie plus agréable $^{19}$. Durant cette décennie, la moitié des départs se dirigeait vers les banlieues de la « zone métropolitaine » de Jérusalem, qui s'étend à l'ouest jusqu'aux villes de Bet Shemesh et Modiin, et à l'est et au sud, jusqu'aux colonies de Maale Adumim et du Gush Etzion. Ces émigrants pouvaient donc continuer à travailler à Jérusalem. Un tiers du total choisissait les colonies cisjordaniennes (Maale Adumim, Betar Illit, Modiin Illit), souvent en raison des avantages financiers proposés par ces localités (moindres coûts immobiliers, aides de l'Etat, faiblesse des taxes locales) et parfois également en raison de motivations idéologiques, encouragées par certains mouvements politiques exerçant une influence sur le gouvernement (les colonies constituant des points de peuplement destinés à appuyer les revendications territoriales de certains mouvements sionistes).

A l'inverse, seuls $12 \%$ de ceux qui partaient vers l'agglomération de Tel Aviv et la plaine côtière mentionnaient la question du logement. En effet, en 2002, un logement de 3 à 4 pièces coûtait en moyenne 1,3 millions de shekels (228 000 euros) à Tel Aviv 
contre 870000 (153 000 euros) à Jérusalem : le différentiel de coût se révélait être plutôt à l'avantage de Jérusalem ${ }^{20}$.

21 Deux profils d'émigrants israéliens peuvent alors être dressés: ceux qui quittent Jérusalem pour des localités de la zone métropolitaine (faisant fonction de «banlieues ») mentionnent dans $68 \%$ des cas le logement comme facteur décisif, et ceux qui partent vers l'agglomération de Tel Aviv et la plaine côtière évoquent l'emploi dans $30 \%$ des cas $^{21}$.

Dans la première catégorie se trouve une proportion importante d'ultra-orthodoxes, si l'on se fie aux destinations choisies par les émigrants (les annuaires statistiques ne présentent pas l'affiliation confessionnelle des émigrants). Parmi les cibles privilégiées se trouvent plusieurs villes habitées majoritairement par des ultra-orthodoxes: Bet Shemesh, Bnei Braq, Betar Illit, Modiin Illit. Les ultra-orthodoxes disposent en moyenne de faibles revenus, et sont plus touchés que les autres catégories sociales par la hausse du coût de l'habitat.

23 La préférence accordée aux banlieues de la zone métropolitaine de Jérusalem et aux villes satellites montre qu'une vaste zone d'influence s'est formée autour de la capitale. Cette mutation s'est accompagnée du déclin du centre-ville ancien et de l'apparition de nouvelles centralités dans les périphéries.

Cette tendance au développement des périphéries de l'agglomération et au déclin du centre-ville ancien ne concerne pas uniquement la population et la ville israéliennes. En effet, la Jérusalem palestinienne connaît une évolution similaire: on observe la formation d'une vaste conurbation de Ramallah à Bethléem, englobant Jérusalem. Pourtant, d'après les statistiques officielles, les flux palestiniens d'entrée et de sortie d'Al Quds seraient de faible ampleur. Il faut donc étudier la nature de ces flux, en ne négligeant pas l'influence des facteurs politiques, notamment des évènements liés au conflit israélo-palestinien et à la recomposition incessante des frontières autour de Jérusalem depuis 1948.

\section{Des flux migratoires palestiniens de faible ampleur?}

Le solde migratoire de la population palestinienne de Jérusalem est négatif au début du $\mathrm{XXI}^{\mathrm{e}}$ siècle. Mais ce solde résulte en tout état de cause de mouvements de faible ampleur $^{22}$. Cela n'a pas toujours été le cas. En effet, la ville a enregistré de fortes pertes migratoires parmi la population palestinienne, à l'occasion de la guerre de 1948, puis après la conquête israélienne de Jérusalem-Est en 1967.

Par ailleurs, nous émettons l'hypothèse que le volume des flux est, aujourd'hui encore, plus conséquent que celui indiqué par les statistiques officielles. Nous pensons qu'une grande partie des flux migratoires s'effectue de manière informelle.

\section{Exode rural versus exil - années 1920 aux années 1960}

Entre 1967 et 2005, la population palestinienne est passée de moins de 70000 à presque 250000 habitants, essentiellement grâce à une vive croissance naturelle, plus qu'au solde migratoire ${ }^{23}$.

28 Néanmoins, l'exode rural en Cisjordanie a alimenté la croissance démographique de Jérusalem. Jusqu'en 1967, les contingents les plus importants provenaient du district 
d'Hébron, voire de la ville même d'Hébron : il s'agissait alors en partie d'exode rural, mais aussi d'une redistribution urbaine, d'une ville moyenne vers la capitale régionale.

Le facteur attractif était le dynamisme économique de Jérusalem, supérieur à celui de la région d'Hébron. Un grand nombre de familles hébronites achetèrent des boutiques dans le souk (marché) de la vieille ville de Jérusalem, ainsi que des logements. Ils acquirent, entre autres, des biens cédés par des familles chrétiennes qui avaient quitté le pays en 1948. Le flux migratoire des Hébronites était entretenu par la vivacité des réseaux de solidarité familiale et communautaire, établis par les premiers arrivés pour accueillir les nouveaux.

Ces migrants ont conservé une identité distincte et ont souffert d'un certain dédain de la part de l'élite traditionnelle de Jérusalem (les Maqdase), parce qu'ils provenaient de zones rurales, que leur niveau d'instruction était souvent inférieur à celui des Hiérosolomitains et qu'un grand nombre étaient de simples commerçants du souk ${ }^{24}$. Aujourd'hui encore, le langage quotidien désigne la deuxième, voire la troisième génération de leurs descendants par le nom de leur lieu d'origine, Khaliliyeh (Hébronites), surnom signifiant qu'ils ne sont pas considérés comme faisant partie intégrante de la population hiérosolomitaine. Pourtant, ils composent au début du $\mathrm{XXI}^{\mathrm{e}}$ siècle environ la moitié de la population arabe de la ville ${ }^{25}$.

\section{Départ des Chrétiens}

31 Avant l'apport massif de l'exode rural, l'élite politique et économique hiérosolomitaine était formée en grande partie de Chrétiens, mais pas exclusivement. Quelques grandes familles musulmanes détenaient également une position sociale confortable, qui avait été acquise sous le régime ottoman et souvent perpétuée sous le régime jordanien. Ces notables servaient de relais entre la population et les gouvernants ${ }^{26}$.

En 2004, ces familles originaires du noyau historique d'Al Quds ne représentent plus que $20 \%$ de la population de l'agglomération. Parmi eux, les Chrétiens sont ceux dont la proportion a le plus diminué : ils ne représentent plus que $5 \%$ de la population totale contre $10 \%$ en 1990. Cette évolution est due à la fois au solde naturel et au solde migratoire. Le taux de fécondité étant beaucoup plus bas parmi les Chrétiens que parmi les Musulmans, la part des Chrétiens s'est donc réduite en valeur relative, comme au Liban par exemple. En outre, le solde migratoire des Chrétiens de Jérusalem est négatif depuis 1948 : un grand nombre a émigré vers l'Europe, l'Amérique, puis plus tard vers les pays du Golfe Persique. Cette élite a été remplacée par les Hébronites, notamment dans le secteur immobilier, le commerce et le tourisme. Les Khaliliyeh tiennent désormais une grande partie du pouvoir économique d'Al Quds.

L'émigration des Chrétiens s'est poursuivie, voire accentuée, après la conquête de Jérusalem-Est par Israël en 1967 et elle perdure jusqu'au début du XXI ${ }^{e}$ siècle. D'après le politologue palestinien Bernard Sabella, plus de 17000 Palestiniens chrétiens de Jérusalem ont émigré à l'étranger entre 1967 et la fin des années 1990. Il y ajoute plus de 7000 Hiérosolomitains qui vivaient déjà à l'étranger en 1967 ou se trouvaient endehors des frontières municipales lors du recensement effectué par l'Etat d'Israël et n'ont de ce fait jamais obtenu le statut de résidant de Jérusalem ${ }^{27}$.

Pourquoi l'émigration touche-t-elle davantage les Chrétiens que les Musulmans? Tout d'abord, l'élite chrétienne dispose des capacités financières nécessaires pour envoyer 
ses descendants étudier à l'étranger : ceux-ci s'installent souvent dans le pays d'accueil où ils trouvent un travail. Ensuite, sa fonction politique et ses aptitudes à l'initiative économique ont été restreintes par la prise de contrôle israélienne de la totalité de l'agglomération, qui s'est manifestée comme un pouvoir politique et économique concurrent. Enfin, l'émigration a elle-même un effet induit sur la fragilisation de la communauté chrétienne restée sur place. Elle devient de plus en plus minoritaire et subit l'influence d'un certain conservatisme dans la société hiérosolomitaine. En quittant le pays, ses membres évitent de se soumettre à la fois à la souveraineté israélienne et à la concurrence de l'élite musulmane montante. Ainsi, la proportion de Chrétiens par rapport à population palestinienne totale est passée de $10 \%$ en 1990 à $5 \%$ en $2001^{28}$.

\section{Des flux modérés?}

$35 \mathrm{Au}$ tournant du XXI ${ }^{\mathrm{e}}$ siècle, le solde migratoire palestinien demeure négatif, mais ces mouvements resteraient modérés : selon l'Annuaire statistique israélien, en 2003, 142 Palestiniens auraient quitté la ville et seuls 3 s'y seraient installés. Ceci est d'autant plus étonnant que l'Annuaire des statistiques emploie le terme d'«Arabes" et non de «Palestiniens » : il devrait donc comptabiliser les Arabes israéliens qui s'installent dans la ville et qui sont sans doute plus nombreux que 3 par an, en sus des Cisjordaniens qui obtiennent le statut de résidant par une procédure de regroupement familial.

Selon l'International Peace and Cooperation Center (centre de recherches palestinien), dans les années 1990 et au début des années 2000, environ 600 à 800 Palestiniens auraient émigré chaque année, tandis que 200 à 600 immigreraient: l'ONG palestinienne confirme un solde migratoire négatif, mais les mouvements sont, selon lui, de plus grande ampleur ${ }^{29}$. Les principales raisons qui poussent les habitants palestiniens à quitter la ville sont similaires à celles qui motivent les départs de leurs voisins israéliens : recherche de logements plus spacieux et à moindre coût et/ou recherche d'un emploi dans la capitale économique de facto (Ramallah pour les Territoires palestiniens, comme Tel Aviv pour Israël), concurrente de la capitale politique, Jérusalem.

37 La validité des données israéliennes concernant les flux migratoires palestiniens demeure contestable, car elles ignorent vraisemblablement l'existence de flux informels de deux sortes. D'une part, une quantité non négligeable de résidents de Jérusalem ont choisi, dans les années 1980 et 1990, de déménager vers les banlieues externes du territoire municipal, tout en conservant une adresse officielle à l'intérieur du périmètre pour préserver leur statut de "résidant de Jérusalem ». Leur nombre est évalué entre 24000 à 60000 personnes selon les sources ${ }^{30}$. La volonté de préserver ce statut tout en s'installant hors de la Jérusalem municipale s'explique par diverses motivations, qu'on ne peut pas catégoriser strictement. D'un point de vue matériel et pragmatique, ce statut permet de bénéficier des subventions sociales israéliennes, d'avoir accès au marché de l'emploi israélien et de pouvoir circuler librement entre la banlieue, espace de résidence, et le cœur de l'agglomération palestinienne, centre commercial, économique et culturel. Mais derrière ces questions matérielles, on peut percevoir également la volonté de conserver un "droit à la ville ", un accès autorisé à Jérusalem à la fois pour les déplacements et pour l'éventualité de s'y installer à nouveau dans le futur. 

Jérusalem ne disposent pas de papiers d'identité en règle. Agnès Levallois et Sophie Pommier estimaient en 1994 que 25000 Palestiniens vivaient en situation irrégulière dans l'agglomération ${ }^{31}$.

Cette estimation est confirmée par les données du Bureau central palestinien des statistiques (PCBS), ou plus précisément, par l'écart entre les données produites par le PCBS et celles produites par l'Institut de Jérusalem pour les études sur Israël (JIIS), qui élabore les statistiques publiques israéliennes. A la fin de l'année 2003, le décalage entre les deux sources au sujet de la population palestinienne totale était de 20570 personnes $^{32}$. D'après l'IPCC, la divergence provient du fait que le PCBS comptabilise comme Hiérosolomitains tous les Palestiniens qui y résident, y compris ceux qui ne détiennent pas de carte de résidant ${ }^{33}$. Cette part importante de résidants illégaux ( $10 \%$ de la population arabe de Jérusalem) prouve que Jérusalem exerce une forte attractivité pour les Palestiniens de Cisjordanie ${ }^{34}$.

Or, des flux et des recompositions se produisent sans nul doute au sein des ces groupes de migrants informels. On peut même supposer que ce type d'habitants est davantage sujet aux fluctuations que les populations en situation régulière, en raison de sa situation précaire. Mais ces flux ne sont pas mesurés par les statistiques officielles.

\section{Regroupement familial : migration cachée?}

Plusieurs observateurs israéliens pensent que certaines demandes de regroupement familial dissimulent une stratégie palestinienne pour migrer des régions de Gaza et Cisjordanie vers Israël, notamment vers Jérusalem-Est ${ }^{35}$. Les services du ministère de l'Intérieur israélien ont en effet constaté que la pratique du «mariages en blanc » a pu être l'une des stratégies à laquelle des résidents des Territoires palestiniens ont parfois recouru pour bénéficier de la nationalité israélienne ou de la carte de résidant de Jérusalem. Cette analyse a été confirmée par des chercheurs indépendants ${ }^{36}$. Légalement, le bénéfice du statut de résidant s'applique au conjoint, et, le cas échéant, aux enfants de celui-ci, même s'ils sont nés d'une union précédente. Certains candidats cisjordaniens ou gazaouites étaient déjà mariés dans les Territoires, mais ont convolé en seconde noce avec une Palestinienne de Jérusalem ou d'Israël, puis ont réclamé une mesure de regroupement familial pour faire venir en Israël les enfants de leur première union ${ }^{37}$. D'après un article publié dans le quotidien israélien Haaretz, cette stratégie vise à faciliter l'accès aux études universitaires et au marché de l'emploi israéliens pour les enfants, voire à faciliter leur émigration vers l'étranger.

En raison de ce soupçon de pratiques frauduleuses, les mesures de regroupement familial des Palestiniens ont été présentées par certains hommes politiques israéliens comme une menace pour l'Etat hébreu. En avril 2002, le ministre de l'Intérieur, Eli Ishaï, membre du parti orthodoxe séfarade Shas, a imposé un gel des regroupements familiaux entre Palestiniens de Cisjordanie-Gaza et Palestiniens d'Israël jusqu'à nouvel ordre. L'arrêté ministériel a été approuvé par le gouvernement le 12 mai $2002^{38}$. Eli Ishaï a exprimé publiquement son inquiétude : d'après lui, si les tendances spontanées se poursuivaient, l'Etat d'Israël perdrait sa majorité démographique juive d'ici 2010, alors qu'il est défini comme Etat juif par la première loi fondamentale, à valeur constitutionnelle ${ }^{39}$. 
seuls 22414 regroupements familiaux ont été acceptés en Israël entre 1993 et 2002 ce qui représente un moyenne de 2500 par an pour l'ensemble du pays ${ }^{40}$. Certes, la quantité de candidats est largement supérieure à celle des dossiers reçus, ce qui pourrait accréditer la thèse de la stratégie d'immigration. Néanmoins, elle reste faible en valeur relative par rapport à la population totale. A Jérusalem, on peut supposer que quelques centaines, voire un millier, ont lieu chaque année. Ce n'est pas négligeable, car chaque regroupement familial peut concerner plusieurs personnes, mais ces procédures ne peuvent en aucun cas être considérées comme une source majeure de flux migratoires ${ }^{41}$.

Les regroupements familiaux réalisés jusqu'en 2002 n'ont donc pas menacé l'identité de l'Etat hébreu. Le démographe Sergio Della Pergola conteste les prévisions alarmistes (d'un point de vue israélien) d'Eli Ishaï ${ }^{42}$. En 2002, à l'ouest de la ligne verte, $80 \%$ des 6,7 millions d'Israéliens étaient Juifs. Sergio Della Pergola évaluait le nombre de nonJuifs parmi les immigrés de l'ancien bloc soviétique à $250000^{43}$. Il estimait qu'Israël accueillait en outre 200000 à 300000 travailleurs étrangers ${ }^{44}$. En revanche, si l'on considérait la population totale, en additionnant celle de Cisjordanie et Gaza à celle qui habite le territoire israélien à l'ouest de la ligne verte, la proportion de Juifs atteignait difficilement la moitié de la population totale. L'espace qui s'étend de la mer Méditerranée à la vallée du Jourdain accueille donc, au début du XXI ${ }^{\mathrm{e}}$ siècle, une proportion à peu près équivalente d'Israéliens juifs et de Palestiniens arabes; mais l'Etat d'Israël, dans ses frontières internationalement reconnues, compte une large majorité d'habitants juifs israéliens.

Néanmoins, les politiques visant à limiter l'immigration de Palestiniens vers Jérusalem et plus généralement vers Israël se sont durcies au tournant du XXI ${ }^{e}$ siècle. Depuis 2002, l'immigration est quasiment impossible. La décision d'établir une frontière physique fermée entre Jérusalem et la Cisjordanie, en 2002, émanait aussi, au-delà des buts sécuritaires affichés, de préoccupations d'ordre démographique. Mais elles n'ont pas empêché l'existence de flux informels, y compris de flux d'émigration hors de Jérusalem, non déclarés par les intéressés afin de conserver une liberté de circulation.

\section{Conclusion}

Les pertes démographiques enregistrées par la ville de Jérusalem, au sein de la population israélienne juive comme de la population palestinienne arabe, traduisent une tendance lourde, installée depuis plusieurs décennies, et ne peuvent être attribuées à une évolution conjoncturelle, qui serait uniquement liée aux épisodes récents du conflit (vagues d'attentat, Intifadas, construction de la barrière de séparation).

Si l'on cherche les causes de cette tendance aux départs, on s'aperçoit tout d'abord que certaines d'entres elles sont communes aux deux populations. A Jérusalem-Ouest comme à Jérusalem-Est, on note l'existence de trajectoires résidentielles délaissant le centre de l'agglomération au bénéfice des périphéries externes, qui s'expliquent principalement par la fuite de coûts immobiliers trop élevés. Ce type de mouvement est classique, il a été observé dans la plupart des grandes villes dans le monde. Il ne signifie pas que la métropole concernée est répulsive : les émigrants continuent souvent à travailler au centre de l'agglomération, et effectuent alors des migrations pendulaires quotidiennes. La zone d'influence de la métropole s'étend, ce qui démontre plutôt la croissance de son attractivité. 

palestiniens à quitter la ville est la recherche d'un emploi. On remarque que cette motivation est à l'origine des départs vers deux bassins d'emploi concurrents de Jérusalem, qui sont l'agglomération de Tel Aviv du côté israélien, et la ville de Ramallah du côté palestinien, devenue la capitale politique (siège du gouvernement) et économique (réceptacle principal des investissements étrangers) depuis le début de l'autonomie palestinienne en 1993. A l'inverse de la précédente, cette motivation traduit un manque d'attractivité de Jérusalem, au moins sur le plan économique.

Toutefois, malgré certaines similitudes quant aux raisons qui alimentent les flux sortants, on note une différence fondamentale au sujet des facteurs qui expliquent la faiblesse des flux entrants à Jérusalem. En effet, les migrations d'Israéliens provenant d'autres régions du pays s'effectuent librement (bien qu'elles puissent être influencées par des facteurs économiques, sociaux, politiques et culturels), tandis que les flux de Palestiniens vers Al Quds ont été d'abord restreints de façon autoritaire par l'Etat hébreu à partir du milieu des années $1990^{45}$, puis rendus quasiment impossibles au début des années 2000, d'une part par la politique de restriction du droit de résidence par le ministère israélien de l'Intérieur, et d'autre part par la construction de la barrière de séparation ${ }^{46}$. On peut donc supposer, dans une certaine mesure, que ces restrictions ont affecté le solde migratoire de la population palestinienne, en d'autres termes, que ce solde aurait pu être positif en l'absence de politiques restrictives. Il ne faut donc pas oublier l'influence du contexte politique pour comprendre le fait migratoire à Jérusalem, même s'il semble parfois difficile de distinguer les différents facteurs explicatifs.

\section{NOTES}

1. Source : Choshen M., Kimhi I., Les dynamiques migratoires à Jérusalem (en hébreu), Jérusalem, the Jerusalem Institute for Israel Studies, 1998; Choshen M., Jerusalem on the Map, Basic Facts and Trends, 1967-1996, Jérusalem, the Jerusalem Institute for Israel Studies, 1998.

2. «Al Quds» est le nom de Jérusalem en arabe.

3. Tous deux sont des organismes gouvernementaux travaillant de façon autonome, en réalisant leurs propres recensements. Le Bureau palestinien des statistiques conduit des recensements dans les Territoires palestiniens et à Jérusalem-Est depuis 1997. Nous utilisons également les données publiées dans l'Annuaire statistique de Jérusalem par le Jerusalem Institute for Israel Studies, et celles fournies par l'International Peace and Cooperation Center, qui sont des centres de recherche indépendants, respectivement israélien et palestinien.

4. Nous appuyons ce propos sur des entretiens informels avec des Israéliens et sur les discours tenus par les intervenants et le public du colloque: "Jérusalem risque-t-elle une crise économique?» (en hébreu), conférence organisée par le Jerusalem Institute for Israel Studies, Jérusalem, Jerusalem Institute for Israel Studies, 15 novembre 2004.

5. Jérusalem comptait 719900 habitants à la fin de l'année 2005, selon l'annuaire statistique de Jérusalem. Cf. Annuaire statistique de Jérusalem 2006-2007, Jérusalem, Jerusalem Institute for Israel Studies, 2007. L'Etat d'Israël comptait 6,99 millions d'habitants à la fin de l'année 2005 et 
7,11 millions d'habitants à la fin de l'année 2006, selon le Central Bureau of Statistics, cf. www.cbs.gov.il. Le Central Bureau of Statistics israélien inclut toute la population qui possède la nationalité israélienne et les résidants palestiniens de Jérusalem, que ces personnes habitent à l'ouest ou à l'est de la ligne verte (ligne de cessez-le feu de 1949 qui marque la séparation entre Jérusalem-Ouest et Jérusalem-Est).

6. Comprenant les villes de Ramat Gan, Ramat HaSharon, Herzliya, Givatayim, Bnei Brak et Petah Tikva, Bat Yam, Holon et Rishon leTsion. Source : ibid.

7. L'Etat hébreu ne dispose pas d'une constitution, mais d'un ensemble de « lois fondamentales » qui ont valeur de constitution et forment, en principe, un préalable à la rédaction d'une constitution. Cf. Klein C., Le système politique d'Israël, Paris, PUF, coll. Que Sais-Je ?, 1983 ; Klein C., La démocratie d'Israël, Paris, Seuil, 1997.

8. La pratique du judaïsme contemporain est divisée entre trois courants principaux: les traditionalistes (ou conservateurs), les libéraux, et les ultra-orthodoxes (haredim en hébreu, dont l'une des mouvances la plus connue est le hassidisme). D'un point de vue statistique, nous reprenons les critères choisis par le démographe Sergio Della Pergola pour identifier la part des ultra-orthodoxes dans la population israélienne: le réseau éducatif auquel se rattachent les foyers (il existe un important réseau scolaire religieux privé), les sondages d'opinion sur l'affiliation religieuse, les résultats électoraux, et le regroupement confessionnel par quartiers à Jérusalem. Cf. Della Pergola S., « Israel's Population and Middle East Normalization : Trends and Challenges», Lezioni dale Scuole estive sul Processo di Pace, Bari, Universita degli Studi di Bari, Centro Interdipartimentale di Ricerche sulla Pace, 2000.

9. Cf. notamment : Benziman U., "On the way to Bnei Brak", Haaretz, 02.10.2005 ; Lis Y., "For Jerusalem mixed jewish areas, cooperation may be the thing", Haaretz, 14.08.2007. Nous citons ici la presse, mais ces représentations sont également exprimées dans des émissions de télévision et radiophoniques.

10. L'« espace métropolitain » de Jérusalem ne correspond à aucun découpage administratif, ses limites sont donc difficiles à déterminer. Les analyses des géographes, économistes, urbanistes et sociologues divergent quant à son étendue. Différents critères ont été établis, comme les migrations pendulaires domicile-emploi, les zones d'achalandise des surfaces commerciales, le recours aux services de niveau supérieur, etc. cf. Choshen M., Kaplan M., Kimhi I., Politiques pour la protection et le développement durable des collines de Jérusalem et des plaines de Judée (en hébreu), Jérusalem, Jerusalem Institute for Israel Studies, 2000, Hasson S., L'organisation municipale dans l'aire métropolitaine de Jérusalem : alternatives conceptuelles (en hébreu), Jérusalem, Jerusalem Institute for Israel Studies, 2001, Hershkovitz S., Rafiah S., Jerusalem : Urban Characteristics and Major Trends in the City's Development, Jérusalem, Municipalité de Jérusalem, Département de la Planification Stratégique, 1997. Nous entendons ici par ce terme l'espace géographique qui entoure Jérusalem (en territoire israélien et en Cisjordanie) et qui subit son influence en termes de migrations démographiques (quotidiennes, temporaires ou définitives).

11. Source : Choshen M., Kimhi I., Les dynamiques migratoires à Jérusalem, op. cit.

12. Della Pergola S., "Israel's Population and Middle East Normalization.. », op. cit. (nous traduisons de l'anglais).

13. Source :Choshen M., Jerusalem on the Map, Basic Facts and Trends, 1967-1996, Jérusalem, the Jerusalem Institute for Israel Studies, 1998, op. cit., p. 43.

14. Source : Annuaire statistique de Jérusalem 2006-2007, op. cit., table VII/1.

15. Il s'agit ici des personnes qui touchent les allocations de chômage. A titre de comparaison, le taux de chômage s'élevait à $8 \%$ à Tel Aviv et $10 \%$ à Haifa la même année. Source : Annuaire statistique de Jérusalem 2006-2007, op. cit., table VII/2 a.

16. Source : Friedman A., "Jerusalem Economy and the Peace Process ", in Ahimeir O., Breger J., Marshall J (eds.), Jerusalem, a City and its Future, Syracuse, Syracuse University Press, Jérusalem, Jerusalem Institute for Israel Studies, 2002, p. 366. 
17. Source : Annuaire statistique de Jérusalem 2006-2007, op. cit., table VII/6.

18. Friedman A., op.cit., p. 372.

19. Source : Choshen M., Kimhi I., Les dynamiques migratoires à Jérusalem (en hébreu), op. cit.

20. Source : Annuaire statistique de Jérusalem 2003, Jérusalem, Jerusalem Institute for Israel Studies, 2004.

21. Source : Choshen M., Kimhi I., op. cit.

22. Moins 142 personnes entre 2002 et 2003. Source : Choshen M., Kimhi I., op. cit.

23. Source: Annuaire statistique de Jérusalem 2006-2007, Jérusalem, Jerusalem Institute for Israel Studies, 2007, Table III/7.

24. Cf. Al Ju'Beh N., «The Social Development of Jerusalem », in Friedman A., Nasrallah R. (ed.), Jerusalem Berlin Forum : Divided Cities in Transition, Jérusalem, Friedrich Ebert Stiftung, International Peace and Cooperation Center, Jerusalem Institute for Israel Studies, 2003.

25. D'après Nazmi Al Ju'beh, ibid.

26. Cf.Khalidi R., L'identité palestinienne : la construction d'une conscience nationale moderne, Paris, La Fabrique, 2003.

27. Bernard Sabella, politologue à l'Université de Béthléem, cité par Malki R., "The Physical Planning of Jerusalem ", in Maoz M., Nusseibeh S., Jerusalem, Points of Friction - and beyond, The Hague, Boston, Kluwer Law International, 2000.

28. Source: Annuaire Statistique de Jérusalem n¹9: 2001, Jerusalem Institute for Israel Studies, 2002.

29. Cf. Equipe de Planification Stratégique de l'IPCC, "Jerusalem Profile ", in Khamaisi R., Nasrallah R. (ed.), The Jerusalem Urban Fabric: Demography, Infrastructures and Institutions, Jérusalem, International Peace and Cooperation Center, 2003, p. 33.

30. 24000 selon le politologue Bernard Sabella, op. cit., 30000 selon l'avocat Muhamad Dahleh, qui est le conseiller juridique du Comité contre le Mur créé par plusieurs localités septentrionales, cf. Dahleh M., "If this Wall is Built», in Palestine Report, 5 novembre 2003, www.palestinereport.org ; 50000 selon la branche de l'ONU chargée des affaires humanitaires, cf. OCHA, «The Jerusalem Barrier Rand Humanitarian Access », Jérusalem, 2003; 60000 selon Rami Nasrallah, directeur de l'ONG palestinienne International Peace and Cooperation Center, cf. Nasrallah R., "The Concept of Separation: the Route of the Wall and its Direct Impact on Jerusalem », in Abu Ghazaleh R., Brooks R., Khamaisi R.et al., The Wall of Annexation and Expansion : its Impact on Jerusalem Area, Jérusalem, IPCC, 2005.

31. Cf. Levallois A., Pommier S., Jérusalem, de la division au partage, Paris, Michalon, 1995, p. 118.

32. D'après l'Annuaire des Statistiques de Jérusalem 2004 du JIIS, 228700 Palestiniens vivaient à Jérusalem en 2003. D'après le PCBS, ils étaient 249270.

33. Equipe de Planification Stratégique de l'IPCC, « Jerusalem Profile », op. cit., p. 20.

34. Cf. Kadiri A., «A Study of Commuting Labor Force from the West Bank to Israel», Middle East Journal, $\mathrm{n}^{\circ} 52$ (4), 1998, p. 17-30.

35. Cf. Gabriel S., Levy D., « Expectations, Information and Migration. The Case of West Bank and Gaza ", Applied Statistics, n²0,1988, p. 1-13.

36. Nadha Shehadah confirme les pratiques de mariage en blanc de Gazaouites avec des citoyennes palestiniennes d'Israël, d'après des enquêtes de terrain : Shehadah N., " Les paradoxes du mariage précoce à Gaza », Etudes Rurales, janvier-juin 2005, nº 173-174, p. 153-182.

37. Cf. ibid.

38. Arrêté $n^{\circ} 1813$. Il a été reconduit chaque année depuis 2002 et quelques « assouplissements » lui ont été apportés, avec des conditions précises. Cf. www.adalah.org.

39. Source : Haaretz, 12 mai 2002.

40. Source : Bureau central israélien des statistiques.

41. Cf. Bishara A., "Constitutional chauvinism », Haaretz, 9 juin 2006. 
42. Cf. Della PergolaS., «Demographic Trends in Israel and Palestine : Prospects and Policy Implications », American Jewish Yearbook 2003, vol. 103, New York, The American Jewish Committee, 2003.

43. Pour des informations plus précises sur l'immigration des ressortissants de l'ancienne URSS en Israël, voir Berthomière W., Israël et l'immigration. Les Juifs d'ex-URSS, acteurs des enjeux territoriaux et identitaires d'Israël, thèse de doctorat de géographie (ss dir. Gildas Simon), Université de Poitiers, janvier 2000.

44. Provenant d'Europe de l'Est, d'Afrique de l'Ouest, et d'Asie du Sud-Est (Philippines en particulier). Cf. Berthomière W., «Nouvelle Intifada, mondialisation et immigration de travailleurs étrangers : une nouvelle réalité sociale pour Israël », Maghreb-Machrek n 177, 2003.

45. Cf. DestremauB., «L'espace en miettes ou l'appropriation identitaire du territoire ", MaghrebMachreck n 150, Paris, 1995. p. 5-18.

46. Khamaisi R., in Abu Ghazaleh R., Brooks R., Khamaisi R.et al, The Wall of Annexation and Expansion : its Impact on Jerusalem Area, Jérusalem, International Peace and Cooperation Center, 2005

\section{RÉSUMÉS}

Contrairement à ce que pourraient laisser penser les valeurs symbolique et politique attribuées à Jérusalem, le solde migratoire de la ville est négatif depuis plusieurs décennies pour la population israélienne comme pour la population palestinienne. De même, contrairement à ce que pourrait laisser penser la désignation de Jérusalem comme capitale de l'Etat hébreu et comme capitale souhaitée par l'Autorité Palestinienne, les facteurs qui expliquent les mouvements migratoires à Jérusalem ne sont pas exclusivement politiques, même si ces derniers exercent une influence importante sur les flux. Ainsi, des facteurs économiques "classiques ", comme le coût de l'immobilier, le chômage, la recherche d'un emploi, jouent un rôle non négligeable dans les dynamiques d'attraction et de répulsion migratoire pour la population de Jérusalem. De plus, deux aires métropolitaines se sont progressivement formées autour de Jérusalem, l'une pour la population israélienne, l'autre pour la population palestinienne : les banlieues de cette aire attirent de plus en plus d'habitants, manifestant un processus d'étalement urbain observé dans la plupart des métropoles dans le monde.

Despite the high symbolic and political values granted to Jerusalem, the city's net migration balance has been negative for several decades, within the Israeli as well as within the Palestinian population. Similarly, despite the fact that Jerusalem is designated as capital city by the Hebrew State, and claimed as capital by the Palestinian Authority, the migration's movements affecting Jerusalem are not exclusively political, even if political grounds may explain part of the flow. Indeed, "classical" economic factors, such as the cost of housing, unemployment and job search, might play a significant role in the dynamics of demographic attraction and repulsion to and from Jerusalem. In addition, two metropolitan areas have gradually developed around Jerusalem, one concerning the Israeli population, the second concerning the Palestinian population. Within these metropolitan areas, suburbs are attracting more and more inhabitants, expressing an urban spread process that nowadays affect most of the metropolitan cities in the world. 
INDEX

Mots-clés : Jérusalem, conflit israélo-palestinien, solde migratoire, bataille démographique

Keywords : Jerusalem, Israeli-Palestinian conflict, migration balance, demographic struggle 